\title{
SCALAR AND MATRIX COMPLEX NONOSCILLATION CRITERIA
}

\author{
by H. C. HOWARD \\ (Received 13th December 1971)
}

\section{Introduction}

In his recent book on ordinary differential equations Hille (3) devotes a chapter to complex oscillation theory. Drawing upon his own work in this area and the work of Nehari, Schwarz, Taam, and others, he gives a variety of oscillation and nonoscillation theorems for solutions of the differential equation

$$
W^{\prime \prime}(z)+p(z) W(z)=0 ;
$$

where $z$ is a complex variable and $p$ is regular in some appropriate domain. There are a number of results for (1.1) with an arbitrary coefficient $p$ and some discussions for special cases of classical interest, such as the Bessel and Mathieu equations. There is a bibliography at the end of the chapter. For other recent work in this area attention is directed to papers by Herold $(1,2) \operatorname{Kim}(4,5)$ and Lavie (6) where other references are given.

I thank the referee for a number of comments which have improved and clarified this paper.

Here attention is limited to nonoscillation theorems with results first obtained for solutions of (1.1). A generalisation to the case in which (1.1) is a matrix equation, as well as the case of a coupled system of matrix equations, then follows from the techniques used. The word " nonoscillation" is defined precisely in the theorems below. We begin by considering equation (1.1).

\section{The scalar case}

Consider equation (1.1). Let $D$ denote a domain of the $z$ plane in which the solutions of (1.1) are regular (we assume $p$ is regular in $D$ ). First we prove a theorem appraising a function involving some solutions of (1.1).

Theorem 2.1. Suppose $y$ is a solution of (1.1) and $\underline{a}$ is a point of $D$. Define $w(z)=-y^{\prime}(z) / y(z)$ and suppose $|w(a)|<\infty$. Let $\Gamma$ be a smooth Jordan arc contained in $D$, starting at $a$. Let $\phi$ be regular function in $D$ such that $|w(a)|<|\phi(a)|$, and such that

for $z \in \Gamma$. Then, for $z \in \Gamma$,

$$
|\phi(z)|-|\phi(a)| \geqq \int_{a_{\Gamma}}^{z}\left\{|p(t)|+\left|\phi^{2}(t)\right|\right\}|d t|
$$

$$
|\phi(z)|>|w(z)|
$$


Proof. Suppose not. The desired result holds initially since $|\phi(a)|>|w(a)|$, so if (2.2) does not hold for all $z \in \Gamma$ there exists a first point $z^{*}$ on $\Gamma$ such that $\left|\phi\left(z^{*}\right)\right|=\left|w\left(z^{*}\right)\right|$, while

$$
|\phi(z)| \geqq|w(z)|
$$

holds for $z \in \Gamma, z$ " between " $\underline{a}$ and $z^{*}$, with equality only at $z=z^{*}$. We can write, therefore,

$$
\begin{aligned}
\left|\phi\left(z^{*}\right)\right|-|\phi(a)| & \geqq \int_{a_{r}}^{z^{*}}\left\{|p(t)|+\left|\phi^{2}(t)\right|\right\}|d t| \\
& >\int_{a_{r}}^{z^{*}}\left\{|p(t)|+\left|w^{2}(t)\right|\right\}|d t| \\
& \geqq \int_{a_{r}}^{z^{*}}\left|w^{\prime}(t)\right||d t| .
\end{aligned}
$$

The last inequality comes from recalling $w=-y^{\prime} / y$ so $w^{\prime}=p+w^{2}$, as a straightforward calculation shows. Thus

which implies

$$
\begin{aligned}
\left|\phi\left(z^{*}\right)\right|-|\phi(a)| & >\left|\int_{a_{\Gamma}}^{z^{*}} w^{\prime}(t) d t\right| \\
& =\left|w\left(z^{*}\right)-w(a)\right| \\
& \geqq\left|w\left(z^{*}\right)\right|-|w(a)|
\end{aligned}
$$

$$
\left|\phi\left(z^{*}\right)\right|-\left|w\left(z^{*}\right)\right|>|\phi(a)|-|w(a)|>0,
$$

a contradiction to the definition of $z^{*}$. This proves the theorem.

We now prove a nonoscillation theorem for some solutions of (1.1).

Theorem 2.2. Let the notation and hypotheses of Theorem 2.1 hold. Then the solution $y$ of (1.1) mentioned there is such that $y(z) \neq 0$ for $z \in \Gamma$; that is, $y$ is a nonoscillatory solution on $\Gamma$.

Proof. Suppose not. Then there exists a first point $z_{1}$, say, on $\Gamma$ such that $y\left(z_{1}\right)=0$. As $z \rightarrow z_{1}, z \in \Gamma,|w(z)|=\left|-y^{\prime}(z) / y(z)\right| \rightarrow \infty$ because the zeros of nontrivial solutions of (1.1) are simple. But Theorem (2.1) tells us $|w(z)|<|\phi(z)|$ for $z \in \Gamma$. Thus as $z \rightarrow z_{1},|w| \rightarrow \infty$ while $|\phi|$ remains bounded. This contradiction proves the result.

These results can be generalised.

Theorem 2.3. Suppose $y$ is a solution of (1.1) and $\underline{a}$ and $b$ are points of $D$. Define $w(z)$ by

$$
\begin{aligned}
\frac{w(z)+h(z)}{g(z)} & \equiv \frac{\cdot w(z)}{g(z)}+\frac{1}{g(z)} \cdot \int_{b_{\Lambda}}^{z}\left\{g(t) p(t)-\frac{1}{4} \frac{\left(g^{\prime}(t)\right)^{2}}{g(t)}\right\} d t \\
& =-y^{\prime}(z) / y(z)
\end{aligned}
$$


and suppose $|w(a)|<\infty$ (here $g$ is a regular function in $D$ such that $g(z) \neq 0$ for $z \in \Gamma, \Gamma_{0}, \Lambda$, with $\Gamma, \Gamma_{0}, \Lambda$ Jordan arcs in $D, \Lambda=\Gamma \cup \Gamma_{0}, \Gamma_{0}$ a fixed Jordan arc in $D$ joining $b$ to $a$ ). Let $\phi$ be a regular function in $D$ such that $|\phi(a)|>|w(a)|$,

$$
|\phi(z)|-|\phi(a)| \geqq \int_{a_{\mathbf{r}}}^{z} \frac{1}{|g(t)|}\left\{|\phi(t)|+\left|\frac{1}{2} g^{\prime}(t)+h(t)\right|\right\}^{2}|d t|
$$

for $z \in \Gamma$. Then, for $z \in \Gamma$,

$$
|\phi(z)|>|w(z)| .
$$

Proof. The reasonings are parallel to those in Theorem 2.1, and are therefore abbreviated. One need only note that,

$$
\begin{aligned}
|\phi(z)|-|\phi(a)| & \geqq \int_{a_{\Gamma}}^{z} \frac{1}{|g|}\left\{|\phi|+\left|\frac{1}{2} g^{\prime}+h\right|\right\}^{2}|d t| \\
& >\int_{a_{\Gamma}}^{z}\left|\frac{1}{g}\left\{w+\frac{1}{2} g^{\prime}+h\right\}^{2}\right||d t| \\
& =\int_{a_{\Gamma}}^{z}\left|w^{\prime}(t)\right||d t|,
\end{aligned}
$$

since the substitution (2.7) transforms (1.1) into the Riccati equation

$$
w^{\prime}=\frac{1}{g}\left(w+\frac{1}{2} g^{\prime}+h\right)^{2} .
$$

The desired conclusion follows as before.

This theorem leads at once to a nonoscillation theorem, whose proof is obvious, and which is stated below.

Theorem 2.4. Let the notation and hypotheses of Theorem 2.3 hold. Then the solution $y$ of (1.1) mentioned there is nonoscillatory on $\Gamma$.

By simple changes in the preceding calculations another nonoscillation theorem can be obtained. A preliminary lemma will be useful.

Lemma 2.5. Suppose $\phi$ and $w$ are regular functions of $z$ in a domain $D$. Let the line segment $\Gamma=\left\{z: z=a+\rho e^{i \psi}, 0 \leqq \rho \leqq \rho_{0}, \rho_{0}>0,0 \leqq \psi<2 \pi\right\}$ also belong to $D$. If

$$
|\Phi| \cos (\psi+\arg \Phi)>|W| \cos (\psi+\arg W)
$$

where $\Phi=\phi_{1} / \phi_{0}, W=w_{1} / w_{0}, \phi_{i}=\phi^{(i)}(a), w_{i}=w^{(i)}(a)$, with $\left|\phi_{0}\right|=\left|w_{0}\right| \neq 0$, then for $z \in \Gamma, z \neq a$ but sufficiently close to $a$,

$$
|\phi(z)|>|w(z)| \text {. }
$$


Proof. For $z$ on $\Gamma$ and sufficiently close to $\underline{a}$ we have

$$
\begin{aligned}
\left|\frac{\phi(z)}{w(z)}\right| & =\frac{\left|\phi_{0}\right|}{\left|w_{0}\right|}\left\{\frac{\left|1+\phi_{1} / \phi_{0} \rho e^{i \psi}+O\left(\rho^{2}\right)\right|}{\left|1+w_{1} / w_{0} \rho e^{i \psi}+O\left(\rho^{2}\right)\right|}\right\} \\
& =\left(\frac{1+2|\Phi| \rho \cos (\psi+\arg \Phi)+|\Phi \rho|^{2}+O\left(\rho^{2}\right)}{1+2|W| \rho \cos (\psi+\arg W)+|W \rho|^{2}+O\left(\rho^{2}\right)}\right)^{\frac{1}{2}} \\
& =1+\rho\{|\Phi| \cos (\psi+\arg \Phi)-|W| \cos (\psi+\arg W)\}+O\left(\rho^{2}\right) \\
& >1,
\end{aligned}
$$

using (2.11), if $\rho$ be sufficiently small. This proves the result.

The desired theorem will come easily from the next theorem below.

Theorem 2.6. Suppose $y$ is a solution of (1.1) and suppose $\underline{a}$ and $b$ are points of $D$. Define $w(z)$ as in (2.7) and suppose $|w(a)|<\infty$. Suppose $\phi$ is a regular function in D such that $|\phi(a)|=|w(a)|$,

$$
|\phi(a)|-|\phi(z)| \geqq \int_{a_{\Gamma}}^{z} \frac{1}{|g|}\left\{|\phi|+\left|\frac{1}{2} g^{\prime}+h\right|\right\}^{2}|d t|,
$$

and such that (2.11) holds. Then, for $z \in \Gamma$,

$$
|\phi(z)|>|w(z)| \text {. }
$$

Proof. The proof is almost identical with the proof of Theorem 2.3, except Lemma 2.5 is used to ensure that $|\phi(z)|>|w(z)|$ initially on $\Gamma$. Then one has, if $z^{*}$ is the first point on $\Gamma$ where $|\phi(z)|=|w(z)|$,

so

$$
\begin{aligned}
|\phi(a)|-\left|\phi\left(z^{*}\right)\right| & >\left|w\left(z^{*}\right)-w(a)\right| \\
& \geqq-\left|w\left(z^{*}\right)\right|+|w(a)|
\end{aligned}
$$

$$
\left|w\left(z^{*}\right)\right|-\left|\phi\left(z^{*}\right)\right|>|w(a)|-|\phi(a)|=0,
$$

a contradiction. The result follows.

It is now obvious we can prove a nonoscillation theorem similar to Theorem 2.4. We have the following theorem:

Theorem 2.7. Let the notation and hypotheses of Theorem 2.6 hold. Then the solution y mentioned there is nonoscillatory on $\Gamma$.

Proof. The proof is obvious.

The techniques used above can be easily modified to apply to a system of differential equations. Consider the system

$$
\begin{aligned}
u^{\prime}(t) & =e(t) v \\
v^{\prime}(t) & =-f(t) u
\end{aligned}
$$

where $t$ is a complex variable and $e$ and $f$ are assumed to be regular in a domain $D$ of the $t$ plane where solutions of (2.14) are also regular. Except for certain 
changes in the calculations the reasonings in the present context are analogous to reasonings of previous theorems so we merely state a nonoscillation theorem pertaining to system (2.14).

Theorem 2.8. Let $(u, v)$ be a solution vector to system (2.14). Suppose $\Gamma$ and $\Lambda$ are smooth Jordan arcs in the domain $D$ of the $t$ plane mentioned above, and let $\underline{a}$ and $b \in D$. Assume $e \neq 0$ on $\Gamma$ and $\Lambda$. Define $w(t)$ by

$$
\frac{w(t)+h(t)}{g(t)} \equiv \frac{w(t)+\int_{b_{\Lambda}}^{t}\left\{g(s) f(s)-\frac{1}{4} \frac{\left(g^{\prime}(s)\right)^{2}}{g(s) e(s)}\right\} d s}{g(t)}=-v(t) / u(t)
$$

and assume $|w(a)|<\infty$. Suppose there exists a function $\phi$, regular in $D$, such that $|\phi(a)|>|w(a)|$ and

$$
|\phi(z)|-|\phi(a)| \geqq \int_{a_{\Gamma}}^{z}\left|\frac{e}{g}\right|\left\{|\phi|+\left|\frac{1}{2}\left(g^{\prime} \mid e\right)+h\right|\right\}^{2}|d s|
$$

for $z$ on $\Gamma$. Here $g$ is a regular function in $D$ which is $\neq 0$ on $\Gamma, \Gamma_{0}, \Lambda$ as in Theorem 2.3. Then the first component of the solution vector $(u, v)$ given above is nonoscillatory on $\Gamma$.

It may be noted it would be no harder to prove a nonoscillation theorem for the second component $v$ of the solution vector. Furthermore, except for the extra notation, one could consider the full linear system

$$
\begin{aligned}
u^{\prime} & =a(t) u+e(t) v \\
v^{\prime} & =-f(t) u+b(t) v
\end{aligned}
$$

just as easily. Finally, if one makes the initial substitution

$$
\frac{w(t)}{g(t)}=-v(t) / u(t)
$$

(with $g$ as above) the Riccati equation associated with (2.14) is

$$
w^{\prime}=\frac{e w^{2}}{g}+\left(g^{\prime} / g\right) w+g f,
$$

so if there exists a regular function $\phi$ such that $|\phi(a)|>|w(a)|$ and

$$
|\phi(t)|-|\phi(a)| \geqq \int_{a_{\Gamma}}^{t}\left\{\left|\frac{e}{g} \phi^{2}\right|+\left|\frac{g^{\prime} \phi}{g}\right|+|g f|\right\}|d s|
$$

a nonoscillation theorem follows without the hypothesis $e \neq 0$ on $\Gamma$ and $\Lambda$.

We shall apply these theorems in a later section.

\section{The matrix case}

We begin by considering the equation

$$
Y^{\prime \prime}(z)+P(z) Y(z)=0,
$$

where $P$ and $Y$ are $(n \times n)$ matrices and the entries in $P$ are regular functions of 
$z$ in a common domain $D$. It is assumed $D$ is such that the components $y_{i j}(z)$ of $Y$ are also regular in $D$. The following lemma will be useful in obtaining nonoscillation theorems.

Lemma 3.1. Let $\Phi(z)$ be an $(n \times n)$ scalar matrix $\Phi(z)=\phi(z) I$ over the complex field. Let $x$ be an $(n \times 1)$ complex column vector of unit length, $\|x\|=1\left(\right.$ where $\left.(x, y) \equiv \sum_{1}^{n} x_{i} \bar{y}_{i},\|x\|^{2} \equiv(x, x)\right)$. Then if W(z) is a $(n \times n)$ matrix over the complex field such that

$$
\frac{|(\Phi(z) x, x)|}{\sqrt{ } 2}>|(W(z) x, x)|
$$

for all $x$ such that $\|x\|=1$ then

$$
\left|\left(\Phi^{2}(z) x, x\right)\right|>\left|\left(W^{2}(z) x, x\right)\right| .
$$

Proof. Let $|(\Phi(z) x, x)| \equiv|\phi(z)|=R_{0} ;$ let $\max _{\|x\|=1}|(W(z) x, x)|=R_{1}$. Then by hypothesis

$$
R_{0}>\sqrt{ } 2 R_{1} \text {. }
$$

Since $R_{1} \geqq|(W(z) x, x)|,\|x\|=1$ we conclude

$$
-R_{1} \leqq \lambda_{1} \leqq(H(z) x, x) \leqq \lambda_{n} \leqq R_{1}
$$

and

where

$$
-R_{1} \leqq \mu_{1} \leqq(K(z) x, x) \leqq \mu_{n} \leqq R_{1},
$$

$$
W(z) \equiv H(z)+i K(z)
$$

with

$$
H(z) \equiv \frac{W(z)+W^{*}(z)}{2}, \quad K(z)=\frac{W(z)-W^{*}(z)}{2 i},
$$

and $H$ and $K$ are hermitian matrices with eigenvalues $\lambda_{1} \leqq \ldots \leqq \lambda_{n}$ and $\mu_{1} \leqq \ldots \leqq \mu_{n}$ respectively. We now write

$$
W^{2}=H^{2}-K^{2}+i(H K+K H)
$$

so

$$
\left.\left|\left(W^{2}(z) x, x\right)\right|^{2}=\left\{\left(H^{2} x, x\right)\right\}-\left(K^{2} x, x\right)\right\}^{2}+\{(H K x, x)+(K H x, x)\}^{2} .
$$

Expanding the right side of (3.8) gives

$$
\left(H^{2} x, x\right)^{2}-2\left(H^{2} x, x\right)\left(K^{2} x, x\right)+\left(K^{2} x, x\right)^{2}+\{2 \operatorname{Re}(K x, H x)\}^{2},
$$

since $H$ and $K$ are hermitian matrices and $(H K x, x)=\left(K x, H^{*} x\right)=(K x, H x)$ while $(K H x, x)=(H x, K x)=(\overline{K x, H x})$. But

$$
\begin{aligned}
4 \cdot \operatorname{Re}^{2}(K x, H x) \leqq 4|(K x, H x)|^{2} \leqq 4\|K x\|^{2}\|H x\|^{2}=4(H x, H x)(K x, K x) \\
=4\left(H^{2} x, x\right)\left(K^{2} x, x\right),
\end{aligned}
$$


so (3.8) becomes

Hence

$$
\begin{aligned}
\left|\left(W^{2}(z) x, x\right)\right|^{2} & \leqq\left(H^{2} x, x\right)^{2}+2\left(H^{2} x, x\right)\left(K^{2} x, x\right)+\left(K^{2} x, x\right)^{2} \\
& =\left\{\left(H^{2} x, x\right)+\left(K^{2} x, x\right)\right\}^{2} \\
& \leqq\left(\max \lambda_{i}^{2}+\max \mu_{j}^{2}\right)^{2} \\
& \leqq\left(R_{1}^{2}+R_{1}^{2}\right)^{2}=4 R_{1}^{4}
\end{aligned}
$$

by (3.3) we get

$$
\left|\left(W^{2}(z) x, x\right)\right| \leqq 2 R_{1}^{2}
$$

$$
\left.\mid W^{2}(z) x, x\right)\left.\left|\leqq 2 R_{1}^{2}<R_{0}^{2}=\right| \phi(z)\right|^{2}=\left|\left(\Phi^{2}(z) x, x\right)\right|,
$$

the desired result.

We next prove a comparison theorem for some of the solutions of (3.1).

Theorem 3.2. Suppose $\Gamma$ is a smooth Jordan arc in D starting at a. Suppose $\Phi(z)$ is a scalar matrix, $\Phi(z)=\phi(z) I$ such that, for any unit column vector $x$,

and

$$
\frac{|(\Phi(z) x, x)|}{\sqrt{ } 2}-\frac{|(\Phi(a) x, x)|}{\sqrt{ } 2} \geqq \int_{a_{r}}^{z}\left\{\left|\left(\Phi^{2}(t) x, x\right)\right|+|(P(t) x, x)|\right\}|d t|
$$

$$
\frac{|(\Phi(a) x, x)|}{\sqrt{ } 2}>|(U(a) x, x)|
$$

where $U(z) \equiv-Y^{\prime}(z) Y^{-1}(z)$ and $Y$ is a solution of (3.1) initially invertible on $\Gamma$. Here we assume also $\phi$ is regular in $D$. Then

$$
\frac{|(\Phi(z) x, x)|}{\sqrt{ } 2}>|(U(z) x, x)| \quad \text { for } z \in \Gamma .
$$

Here the scalar product is the same as in Lemma 3.1, and the appraisals in (3.13) (3.14) are uniform in $x$.

Proof. Suppose the assertion is false. Then there exists a first point, $z^{*}$, say, on $\Gamma$ and a unit vector $x_{0}$ such that

$$
\frac{\left|\left(\Phi\left(z^{*}\right) x_{0}, x_{0}\right)\right|}{\sqrt{2}}=\left|\left(U\left(z^{*}\right) x_{0}, x_{0}\right)\right|
$$

We have, from (3.13),

$$
\begin{aligned}
\frac{\left|\left(\Phi\left(z^{*}\right) x_{0}, x_{0}\right)\right|}{\sqrt{2}-\frac{\left|\left(\Phi(a) x_{0}, x_{0}\right)\right|}{\sqrt{ } 2}} \geqq \int_{a_{\Gamma}}^{z^{*}}\left\{\left|\left(\Phi^{2}(t) x_{0}, x_{0}\right)\right|+\left|\left(P(t) x_{0}, x_{0}\right)\right|\right\}|d t| \\
>\int_{a_{\Gamma}}^{z^{*}}\left\{\left|\left(U^{2}(t) x_{0}, x_{0}\right)\right|+\left|\left(P(t) x_{0}, x_{0}\right)\right|\right\}|d t|
\end{aligned}
$$

(since the appraisal (3.15) holds for all $z$ on $\Gamma$ " between " $a$ and $z^{*}$ and we can 
use Lemma 3.1 to replace the $\Phi^{2}$ term with $U^{2}$ )

$$
\geqq\left|\int_{a_{\Gamma}}^{z^{*}}\left(U^{\prime}(t) x_{0}, x_{0}\right) d t\right|
$$

(since the substitution $U=-Y^{\prime} Y^{-1}$ transforms (3.1) into the matrix Riccati equation $U^{\prime}=U^{2}+P$ )

$$
\begin{aligned}
& =\left|\left(U\left(z^{*}\right) x_{0}, x_{0}\right)-\left(U(a) x_{0}, x_{0}\right)\right| \\
& \geqq\left|\left(U\left(z^{*}\right) x_{0} x_{0}\right)\right|-\left|\left(U(a) x_{0}, x_{0}\right)\right| .
\end{aligned}
$$

Combining the extremes of this chain of inequalities yields

$$
\frac{\left|\left(\Phi\left(z^{*}\right) x_{0}, x_{0}\right)\right|}{\sqrt{ } 2}-\left|\left(U\left(z^{*}\right) x_{0}, x_{0}\right)\right|>\frac{\left|\left(\Phi(a) x_{0}, x_{0}\right)\right|}{\sqrt{ } 2}-\left|\left(U(a) x_{0}, x_{0}\right)\right|>0,
$$

by use of (3.14), a contradiction to the definition of $z^{*}$.

We can now prove a nonoscillation theorem for some solutions of (3.1).

Theorem 3.3. Let the notation and hypotheses of Theorem 3.2 hold. Then determinant $Y(z)=\operatorname{det} Y(z) \neq 0$ for $z \in \Gamma$, which we express by saying $Y$ is a nonoscillatory solution of (3.1) on $\Gamma$.

Proof. Our hypotheses say $Y(z)$ is initially invertible for $z$ on $\Gamma$. Suppose there were a first point $z_{1} \in \Gamma$ such that det $Y\left(z_{1}\right)=0$. This implies

$$
\lim _{z \in \Gamma} \sup _{z \rightarrow z_{1}}\left|\left(-Y^{\prime}(z) Y^{-1}(z) x, x\right)\right| \equiv \lim _{z \in \Gamma} \sup _{z \rightarrow z_{1}}|(U(z) x, x)|=\infty
$$

for at least one unit vector $x=x_{0}$, say. Suppose not. Then there exists a constant $M$ such that

$$
\lim _{z \in \Gamma} \sup _{z \rightarrow z_{1}}|(U(z) x, x)|<M
$$

for all unit vectors $x$. In particular, choosing $x=(1,0, \ldots, 0), x=(0,1,0, \ldots, 0)$, and so on, we see that $\lim _{z \rightarrow \Gamma \rightarrow z_{1}} \sup _{z i i}(z) \mid<M$ if $U(z)=\left(u_{i j}(z)\right), i, j=1,2, \ldots, n$, Thus $\mid$ trace $U(z) \mid$ is bounded as $z \rightarrow z_{1}$ on $\Gamma$ and

$$
\operatorname{det} Y(z) \equiv \operatorname{det} Y(a) e^{\int_{a}^{z} \operatorname{trace}\{-U(t)\} d t}
$$

is bounded away from zero as $z \rightarrow z_{1}$ since det $Y(a) \neq 0$. But det $Y\left(z_{1}\right)=0$, which is a contradiction, and the result follows. Now we use Theorem (3.2) to conclude

$$
\frac{\left|\left(\Phi(z) x_{0}, x_{0}\right)\right|}{\sqrt{2}}>\left|\left(U(z) x_{0}, x_{0}\right)\right|
$$

for $z \rightarrow z_{1}, z \in \Gamma$ an impossibility since the term on the left in (3.20) is bounded, the term on the right unbounded. This proves the theorem.

The next theorem generalises Theorem 3.2. The main ideas are fundamentally the same so the proof is abbreviated. 
Theorem 3.4. Consider the matrix system

$$
\begin{aligned}
& Y^{\prime}(z)=E(z) Z(z) \\
& Z^{\prime}(z)=-F(z) Y(z) .
\end{aligned}
$$

Suppose $D$ is a domain in the $z$ plane where the coefficient matrices $E, F$ and the solution matrices are all regular. Let $\Gamma$ be a smooth Jordan arc in $D$, starting at $\underline{a}$, say. Suppose there exists a scalar matrix $\Phi(z)=\phi(z) I(\phi$ regular in $D)$ such that the following appraisals hold, for $z \in \Gamma$, (uniformly in $x,\|x\|=1$ )

$$
\begin{gathered}
\frac{|(\Phi(z) x, x)|}{2^{z}}-\frac{|(\Phi(a) x, x)|}{2^{z}} \geqq \int_{a_{\Gamma}}^{z}\left\{\left|\left(\Phi^{2}(t) x, x\right)\right| M_{E}(t)+|(F(t) x, x)|\right\}|d t| \\
\frac{|(\Phi(a) x, x)|}{2^{z}}>|(U(a) x, x)|
\end{gathered}
$$

(where $M_{E}(z) \equiv(\alpha(z)+\beta(z)), \alpha(z)=\max _{i}\left|\alpha_{i}(z)\right|, \beta(z)=\max _{i}\left|\beta_{i}(z)\right|, \alpha_{i}, \beta_{i}$ the (real) eigenvalues of $\frac{E(z)+E^{*}(z)}{2}$ and $\frac{E(z)-E^{*}(z)}{2 i}$ with $E$ such that $M_{E}(z) \neq 0$ on $\Gamma$, and $U(z)=-Z(z) Y^{-1}(z), Z$ and $Y$ a solution pair of (3.21) with $Y$ assumed invertible initially for $z \in \mathrm{F})$. Then

$$
\frac{|(\Phi(z) x, x)|}{2^{z}}>|(U(z) x, x)|
$$

for $z \in \Gamma$, and any $x$ such that $\|x\|=1$.

Proof. Suppose the assertion is false. Then there exists a first point, $z^{*}$ say, on $\Gamma$ and a unit vector $x_{0}$, such that

$$
\frac{\left|\left(\Phi\left(z^{*}\right) x_{0}, x_{0}\right)\right|}{2^{z}}=\left|\left(U\left(z^{*}\right) x_{0}, x_{0}\right)\right|
$$

but for any other $z \in \Gamma$ " between " $\underline{a}$ and $z^{*},(3.24)$ holds. Let

$$
|(\Phi(z) x, x)| \equiv|\phi(z)|=R_{0}
$$

( $z$ is fixed now in the following discussion). Let $R_{1}=\max _{\|x\|=1}|(U(z) x, x)|$. Then we have

$$
R_{0}>2^{\frac{3}{2}} R_{1} \text {. }
$$

Since $R_{1} \geqq|(U(z) x, x)|,\|x\|=1$ we conclude

and

$$
-R_{1} \leqq \lambda_{1} \leqq(H(z) x, x) \leqq \lambda_{n} \leqq R_{1}
$$

where

$$
-R_{1} \leqq \mu_{1} \leqq(K(z) x, x) \leqq \mu_{n} \leqq R_{1}
$$

with

$$
U(z) \equiv H(z)+i K(z)
$$

$$
H(z)=\frac{U(z)+U^{*}(z)}{2}, \quad K(z)=\frac{U(z)-U^{*}(z)}{2 i}
$$


and the $\lambda_{i}$ and $\mu_{i}$ are the eigenvalues, respectively of $H$ and $K$. Now write

$$
U E U=(H+i K)(A+i B)(H+i K)
$$

where $E=A+i B, A=\frac{E+E^{*}}{2}, B=\frac{E-E^{*}}{2 i}$, Thus, computing, we have

$$
\begin{aligned}
|(U E U x, x)|^{2}= & \{((H A H x, x)-(K A K x, x))-((K B H x, x)+(H B K x, x))\}^{2} \\
& +\{((H B H x, x)-(K B K x, x))+((K A H x, x)+(H A K x, x))\}^{2} \\
= & \{((H A H x, x)-(K A K x, x))-2 \operatorname{Re}(K B H x, x)\}^{2} \\
& +\{((H B H x, x)-(K B K x, x))+2 \operatorname{Re}(K A H x, x)\}^{2} \\
\leqq & \{(H A H x, x)|+|(K A K x, x)|+2|(K B H x, x) \mid\}^{2} \\
& +\{|(H B H x, x)|+|(K B K x, x)|+2|(K A H x, x)|\}^{2} \\
\leqq & \left\{\alpha(z) \cdot \max _{j} \lambda_{j}^{2}+\alpha(z) \max _{i} \mu_{i}^{2}+2 \beta(z) \max _{j}\left|\lambda_{j}\right| \cdot \max _{i}\left|\mu_{i}\right|\right\}^{2} \\
& +\left\{\beta(z) \cdot \max _{j} \lambda_{j}^{2}+\beta(z) \max _{i} \mu_{i}^{2}+2 \alpha(z) \max _{j}\left|\lambda_{j}\right| \cdot \max _{i}\left|\mu_{i}\right|\right\}^{2} \\
\leqq & \left\{2 \alpha(z) R_{1}^{2}+2 \beta(z) R_{1}^{2}\right\}^{2}+\left\{2 \beta(z) R_{1}^{2}+2 \alpha(z) R_{1}^{2}\right\}^{2} \\
= & 8(\alpha(z)+\beta(z))^{2} R_{1}^{4} .
\end{aligned}
$$

$$
|(U E U x, x)| \leqq 2^{\frac{3}{2}} R_{1}^{2} M_{E}(z)<R_{0}^{2} M_{E}(z)=M_{E}(z)\left|\left(\Phi^{2}(z) x, x\right)\right|
$$

from (3.26). The desired theorem now follows readily from (3.32). We have

$$
\begin{aligned}
\frac{\left|\left(\Phi\left(z^{*}\right) x_{0}, x_{0}\right)\right|}{2^{z}} & -\frac{\left|\left(\Phi(a) x_{0}, x_{0}\right)\right|}{2^{z}} \\
& \geqq \int_{a_{\Gamma}}^{z^{*}}\left\{\left|\left(\Phi^{2}(t) x_{0}, x_{0}\right)\right| M_{E}(t)+\left|\left(F(t) x_{0}, x_{0}\right)\right|\right\}|d t| \\
& >\int_{a_{\Gamma}}^{z^{*}}\left\{\left|\left(U(t) E(t) U(t) x_{0}, x_{0}\right)\right|+\left|\left(F(t) x_{0}, x_{0}\right)\right|\right\}|d t| \\
& \geqq\left|\int_{a_{\Gamma}}^{z^{*}}\left(U^{\prime}(t) x_{0}, x_{0}\right) d t\right|
\end{aligned}
$$

(by use of (3.32))

Thus

(since the substitution $U=-Z Y^{-1}$ leads to the Riccati matrix equation

$$
\begin{aligned}
\left.U^{\prime}=U E U+F\right) & \geqq\left|\left(U\left(z^{*}\right) x_{0}, x_{0}\right)-\left(U(a) x_{0}, x_{0}\right)\right| \\
& \left.\geqq \mid U\left(z^{*}\right) x_{0}, x_{0}\right)|-|\left(U(a) x_{0}, x_{0}\right) \mid .
\end{aligned}
$$

$$
\frac{\left|\left(\Phi\left(z^{*}\right) x_{0}, x_{0}\right)\right|}{2^{\frac{z}{4}}}-\left|\left(U\left(z^{*}\right) x_{0}, x_{0}\right)\right|>\frac{\left|\left(\Phi(a) x_{0}, x_{0}\right)\right|}{2^{\frac{3}{3}}}-\left|\left(U(a) x_{0}, x_{0}\right)\right|>0
$$

a contradiction to $(3.25)$. This proves the result. 
We now have a lemma which is necessary to obtain a nonoscillation theorem.

Lemma 3.5. Let $Y, Z$ be a pair of matrix solutions of (3.21) for $z \in \Gamma, \Gamma$ as in Theorem 3.4. Let $Y$ be invertible for $z \in \Gamma, z$ " between " $a$ and $z_{1}$ say $\left(z_{1} \neq a\right)$, with $Y$ singular first at $z_{1}$. Let $E$ be a symmetric matrix. Then there exists at least one unit vector $x=x_{0}$, say, such that

where $U=-Z Y^{-1}$.

$$
\lim _{z \in \mathbf{r}} \sup _{z \rightarrow z_{1}}\left|\left(U(z) x_{0}, x_{0}\right)\right|=\infty,
$$

Proof. Suppose not. Then there exists a constant $M$ such that

$$
\lim _{z \in \Gamma} \sup _{z \rightarrow z_{1}}|(U(z) x, x)|<M
$$

for all unit vectors $x$. Taking $x=(1,0,0, \ldots, 0), x=(0,1,0, \ldots, 0)$, and so on, we see

$$
\lim _{z \in \Gamma} \sup _{z \rightarrow z_{1}}\left|u_{i i}(z)\right|<M
$$

if $U(z)=\left(u_{i j}(z)\right) i, j=1, \ldots, n$. Moreover, taking $x=(1 / \sqrt{ } 2,1 / \sqrt{ } 2,0, \ldots)$ $x=(1 / \sqrt{ } 2,0,1 / \sqrt{ } 2,0, \ldots, 0)$ and so on, we have

Now we can write

$$
\lim _{z \in \Gamma} \sup _{z \rightarrow z_{1}}\left|u_{i j}(z)+u_{j i}(z)\right|<4 M \text {. }
$$

$$
Y^{\prime} Y^{-1}=E Z Y^{-1}=-E U
$$

so

$$
\operatorname{det} Y(z)=\operatorname{det} Y(a) e^{I} \text { where } I=\int_{a_{\Gamma}}^{z}-\operatorname{trace}(E(s) U(s)) d s
$$

for $z$ on $\Gamma$. The indicated trace is easily computed and we have

$$
\text { trace } E U=\sum_{i=1}^{n}\left\{\sum_{j=1}^{n} e_{i j} u_{j i}\right\} \text {. }
$$

The terms on the right of (3.39) can be broken up into two types, those terms in which $i=j$, and those terms in which $i \neq j$. The latter occur in the form $e_{i j} u_{j i}+e_{j i} u_{i j}$, with $e_{i j}=e_{j i}$. Hence

$$
\begin{aligned}
\mid \text { trace } E U \mid & \leqq \sum_{i=1}^{n}\left|e_{i i}\right| \cdot M+\sum_{i, j=1, i \neq j}^{n}\left|e_{j i}\right|\left\{\left|u_{i j}+u_{j i}\right|\right\} \\
& =M \sum_{i=1}^{n}\left|e_{i i}\right|+4 M \sum_{i, j=1, i \neq j}^{n}\left|e_{j i}\right| .
\end{aligned}
$$

Since $E$ is assumed to be regular in $D$, and hence on $\Gamma$ we have

$$
\mid \text { trace } E(s) U(s) \mid<M_{1}<\infty \text {, say, }
$$

for $s$ on $\Gamma$ between $a$ and $z_{1}$. From (3.38) we conclude det $Y(z)$ is bounded away from zero as $z \rightarrow z_{1}, z \in \Gamma$. This is in contradiction to the assumption det $Y\left(z_{1}\right)=0$ and the desired result follows.

Our desired nonoscillation theorem now follows.

$$
\text { E.M.S. }-M
$$


Theorem 3.6. Suppose the notation and conventions of Theorem 3.4 hold. Suppose $E$ is a symmetric matrix on $\Gamma$. Suppose there exists a matrix $\Phi$ as in Theorem 3.4 such that the appraisals (3.22) and (3.23) hold. Then $Y(z)$ is nonoscillatory on $\Gamma$ in the sense $\operatorname{det} Y(z) \neq 0$.

Proof. Suppose not. Our hypotheses state $Y$ is initially invertible on $\Gamma$. Thus we are assuming there exists a first point $z_{1}$, say, on $\Gamma$ such that $\operatorname{det} Y\left(z_{1}\right)=0$. From Theorem 3.4 we have

$$
\frac{|(\Phi(z) x, x)|}{2^{z}}>|(U(z) x, x)|
$$

$z \in \Gamma$. By Lemma 3.5 there exists at least one unit vector, call it $x_{0}$, such that

$$
\lim _{z \in \Gamma} \sup _{z \rightarrow z_{1}}\left|\left(U(z) x_{0}, x_{0}\right)\right|=\infty .
$$

This clearly contradicts (3.42) since $\Phi$ is regular on $\Gamma$, and (3.42) holds for any unit vector, in particular $x_{0}$. This proves the theorem.

\section{Applications}

We.investigate the nonoscillatory behaviour of solutions of the equation

where we assume:

$$
W^{\prime \prime}(z)+p(z) W(z)=0,
$$

$p$ is singular only at zero and a finite number of other points in the finite

$$
p(z)=\frac{B}{z^{2}} e(z)
$$

for $z$ near zero, with $e(z)$ regular at zero, $e(0)=1$ and $0<B<1 / 4$,

$$
\left|\int_{0_{A}}^{z} t^{2} p(t) d t\right| \leqq B|z|
$$

for $z \in D$, with $D$ a domain of the $z$ plane in which the solutions of (4.1) are regular, and $\Lambda$ a straight line contour between 0 and $z$ contained in $D$. A nontrivial example is afforded by $p(z) \equiv B / z^{2}, D=\{z: z=x+i y, x \notin(-\infty, 0]\}$.

We first try to use Theorem 2.4. We take $\underline{a} \in D$,

$$
\Gamma=\left\{z: z=a+\rho e^{i \psi}, \quad|\psi| \leqq \pi, \quad 0 \leqq \rho \leqq \rho_{0} \leqq \infty\right\}
$$

The case $\rho_{0}<\infty$ can occur if $\Gamma$ tends toward a branch cut, say, used in the construction of $D$. We set

$$
g(z)=z^{2}, \phi(z)=\frac{1}{4}(z-a)
$$

and check if inequality (2.8) holds, with

$$
h(t)=\int_{0_{\wedge}}^{t}\left\{g p-\frac{1}{4}\left(g^{\prime}\right)^{2} / g\right\} d s .
$$


One wants

$$
\frac{1}{4} \rho-0 \geqq \int_{a_{\Gamma}}^{a+\rho e^{i \psi}} \frac{1}{|t|^{2}}\left\{\frac{1}{4}|t-a|+\left|t+\int_{0_{\Lambda}}^{t} s^{2} p(s) d s-\int_{0_{\Lambda}}^{t} d s\right|\right\}^{2}|d t| .
$$

But $\left|t-t+\int_{0}^{t} s^{2} p(s) d s\right| \leqq B|t|$ by (4.4) so it is sufficient to verify the more stringent inequality

If $a=\alpha+i \beta$ we have

$$
\frac{1}{4} \rho \geqq \int_{0}^{\rho}\left\{\frac{\rho}{\frac{\rho}{\left|a+\rho e^{i \psi}\right|}}+B\right\}^{2} d \rho .
$$

$$
\frac{\rho}{\left|a+\rho e^{i \psi}\right|}=\frac{\rho}{\left\{|a|^{2}+2|a| \rho \cos (\psi-\phi)+\rho^{2}\right\}^{\frac{1}{2}}}
$$

where $\tan \phi=\beta / \alpha . \quad$ If $\cos (\psi-\phi) \geqq 0$ we have $\frac{\rho}{\left|a+\rho e^{i \psi}\right|} \leqq 1$ so (4.8) is true if

$$
\frac{1}{4} \rho \geqq \int_{0}^{\rho}\left\{\frac{1}{4}+B\right\}^{2} d \rho=\left(\frac{1}{4}+B\right)^{2} \rho .
$$

Since $1 / 4>B$ by assumption we conclude (4.7) holds if $\cos (\psi-\phi) \geqq 0$. Since $\phi(a)=0$ we must have $w(a)=\left(-y^{\prime}(a) / y(a)\right) a^{2}-h(a)=0$ and to ensure $|\phi(z)|>|w(z)|$ for $z$ on $\Gamma$ and near $\underline{a}$ we demand $\left|\phi^{\prime}(a)\right|>\left|w^{\prime}(a)\right|$. This requires

which will hold if

$$
\frac{1}{4}>\left|\frac{1}{a^{2}}(w(a)+a+h(a))^{2}\right|
$$

$$
\frac{1}{4}>\frac{1}{|a|^{2}}|a+h(a)|^{2} \text {. }
$$

But $|a+h(a)| \leqq B|a|$ and we have taken $B<1 / 4$ so (4.11) also holds. We have the following theorem.

Theorem 4.1. Suppose the notation and assumptions of (4.1), (4.2) and (4.4) (with $0<B<1 / 4$ ) hold. Then, associated with any point $\underline{a} \in D$ there is a solution $y$ of (4.1) such that $y(z) \neq 0$ for $z \in \Gamma$.

By letting $\psi$ vary (compare the definition of $\Gamma$ ) zero free regions for $y$ can be obtained. Roughly speaking, the zero free region for the solution mentioned in Theorem 4.1 consists of a half plane $P$ with boundary line perpendicular to $\arg a$ (recall $|\psi-\phi| \leqq \pi / 2)$ minus the parts of $D$ " shadowed "by the branch cuts used in the construction of $D$, if a light source were placed at $\underline{a}$. Compare this result with Hille (3, Theorem 11.2.8).

We next show how, under certain circumstances, the zero free region can be extended beyond the " half" plane $P$. For the sake of simplicity we assume $\underline{a} \in D$ is a positive real number. Consider

$$
I \equiv \int_{0}^{\rho}\left\{\frac{\rho}{4} \frac{\rho}{\left|a+\rho e^{i \psi}\right|}+B\right\}^{2} d \rho .
$$


One has

$$
\sqrt{ } I \leqq\left(\int_{0}^{\rho} \frac{1}{16} \frac{\rho^{2}}{\left(a^{2}+2 a \rho \cos \psi+\rho^{2}\right)} d \rho\right)^{\frac{1}{2}}+\left(\int_{0}^{\rho} B^{2} d \rho\right)^{\frac{1}{2}}
$$

by the Minkowski inequality. Suppose we assume at this point the angle $\psi$ used in the definition of $\Gamma$ satisfies $\pi / 2 \leqq|\psi| \leqq 3 \pi / 4$. From (4.13) we get, under these circumstances,

$$
\sqrt{ } I \leqq \sqrt{ }\left(\frac{1}{16} \cdot 2 \rho\right)+\sqrt{ }\left(B^{2} \rho\right)
$$

so

$$
I \leqq\left(\sqrt{ } \frac{1}{8}+B\right)^{2} \rho .
$$

Then inequality (2.8) will hold if

$$
\left(\sqrt{ } \frac{1}{8}+B\right)^{2} \leqq \frac{1}{4}
$$

or

$$
B \leqq \frac{1}{2}-\frac{1}{2 \sqrt{ } 2} .
$$

If one takes $\phi, g$ and initial conditions as in Theorem 4.1 (and uses Theorem 4.1 for $|\psi| \leqq \pi / 2$ ) we get the following theorem.

Theorem 4.2. If hypotheses (4.1)-(4.4) hold, except $0<B<\frac{1}{2}-\frac{1}{2} \sqrt{ } 2$, then associated with any positive real number $\underline{a} \in D$ there is a solution $y$ of (4.1) such that $y(z) \neq 0$ on $\Gamma$. The angle $\psi$ associated with the definition of $\Gamma$ is restricted by $|\psi| \leqq 3 \pi / 4$.

At the expense of restricting the location of singularities of $p$ one can get an even larger zero free region. Suppose the domain $D$ is the $z$-plane cut along the negative real axis. From (4.3) and an application of regular singular point theory we know there exists a pair of linearly independent solutions of (4.1) which in the neighbourhood of zero, behave like

where

$$
z^{\alpha_{1}} \text { and } z^{\alpha_{2}}
$$

$$
\alpha_{1}=\frac{1+\sqrt{ }(1-4 B)}{2}, \quad \alpha_{2}=\frac{1-\sqrt{ }(1-4 B)}{2} .
$$

We consider that solution behaving like $z^{\alpha_{1}}$. One has

$$
\begin{aligned}
y_{1}(z) & =z^{a_{1}}\left(1+a_{1} z+\ldots\right) \\
& =z^{\alpha_{1}} S(z), \text { say },
\end{aligned}
$$

where $S(z)$ is regular at zero. Computing we get

$$
\frac{y_{1}^{\prime}(z)}{y_{1}(z)}=\frac{\alpha_{1} z^{\alpha_{1}-1}\left(1+a_{1} z+\ldots\right)+z^{\alpha_{1}}\left(a_{1}+\ldots\right)}{z^{\alpha_{1}}\left(1+a_{1} z+\ldots\right)} .
$$


Near $z=0$, at $z=a$ say, where $|a|$ is small, we get

$$
\begin{aligned}
\frac{y_{1}^{\prime}(a)}{y_{1}(a)} & =\frac{\alpha_{1}}{a}+\frac{\left(a_{1}+\ldots\right)}{\left(1+a_{1} a+\ldots\right)} \\
& =\frac{\alpha_{1}}{a}+a_{1}+O(a) .
\end{aligned}
$$

We have, applying Theorem 2.4

so

$$
\begin{aligned}
w(a) & =\left(-y_{1}^{\prime}(a) / y_{1}(a)\right) a^{2}-h(a) \\
& =-\alpha_{1} a-(B-1) a+O\left(a^{2}\right)
\end{aligned}
$$

$$
\begin{aligned}
|w(a)| & =\left|1-\alpha_{1}-B+O(a)\right| \cdot|a| \\
& =\left|\alpha_{2}-B+O(a)\right| .|a| .
\end{aligned}
$$

We choose $\phi(z)=\left(\frac{1}{4}\right) z$ and get $|\phi(a)|=\left(\frac{1}{4}\right)|a|$. We want

$$
|w(a)|<|\phi(a)|=\left(\frac{1}{4}\right)|a| \text {. }
$$

Suppose $B=\frac{1}{4}-\gamma^{2}, 0<\gamma<\frac{1}{2}$. Then

$$
\alpha_{2}-B=\frac{1-\sqrt{ }(1-4 B)}{2}-B=\frac{1-2 \gamma}{2}-\left(\frac{1}{4}-\gamma^{2}\right)=\frac{1}{4}-\gamma+\gamma^{2}<\frac{1}{4} .
$$

Hence for any $\underline{a}$ such that $|a|$ is sufficiently small we have

We need to verify also

$$
|w(a)|<|\phi(a)| \text {. }
$$

$$
\left|\frac{1}{4}\left(\rho e^{i \psi}+a\right)\right|-\frac{1}{4}|a| \geqq \int_{0}^{\rho} \frac{1}{|t|^{2}}\left(\frac{1}{4}|t|+B|t|\right)^{2} d \rho .
$$

This will be true if $\arg a=\psi$ since then

$$
\left|\frac{1}{4}\left(\rho e^{i \psi}+a\right)\right|=\frac{1}{4}|\rho+| a||
$$

and the left side of (4.25) becomes $\frac{1}{4} \rho$, while the right side is $\left(\frac{1}{4}+B\right)^{2} \rho<\frac{1}{4} \rho$. We have the following theorem.

Theorem 4.3. If hypotheses (4.2) and (4.3) hold, if the domain $D$ is the z plane cut along the negative real axis, and if $a=|a| e^{i \psi}$ has a sufficiently small modulus, then (4.1) possesses a solution $y$ such that $y(z) \neq 0$ on

$$
\Gamma=\left\{z: z=(|a|+\rho) e^{i \psi}, 0 \leqq \rho<\infty,|\psi|<\pi\right\} .
$$

If $|a|$ is sufficiently small we clearly have the same solution $y$ as $\psi$ goes from $-\pi$ to $\pi$ and the theorem allows us to conclude that there exists a solution zero free in $D$, the cut plane.

As a final application we consider Theorem 3.6. As a sample of what results can be obtained we take the fourth order equation

$$
\left(R_{2}(z) y^{\prime \prime}(z)\right)^{\prime \prime}+\left(R_{1}(z) y^{\prime}(z)\right)^{\prime}+R(z) y^{\prime}(z)+R_{0}(z) y(z)=0
$$


and restrict attention to a domain $D$ where the $R_{i}$ as well as the solutions of (4.24) are regular. Consider the two solutions $y_{1}$ and $y_{2}$ of (4.24) such that, if $\underline{a} \in D$,

and

$$
\begin{aligned}
& y_{1}(a)=1, y_{1}^{\prime}(a)=0, y_{1}^{\prime \prime}(a)=0 \\
& \left(R_{2}(z) y_{1}^{\prime \prime}(z)\right)_{z=a}^{\prime}+R_{1}(a) y_{1}^{\prime}(a)=0
\end{aligned}
$$

$$
\begin{aligned}
& y_{2}(a)=0, y_{2}^{\prime}(a)=1, y_{2}^{\prime \prime}(a)=0 \\
& \left(R_{2}(z) y_{2}^{\prime \prime}(z)\right)_{z=a}^{\prime}+R_{1}(a) y_{2}^{\prime}(a)=0 .
\end{aligned}
$$

Except for the " perturbation " $R \boldsymbol{y}^{\prime}$ equation (4.24) is of the type considered earlier by a number of authors $(7,8)$. In particular, by following the calculations of $(8$, pp. $401-403)$ one finds the $(2 \times 2)$ matrices

$$
Y(z) \equiv\left[\begin{array}{rr}
1 & -z \\
0 & 1
\end{array}\right]\left[\begin{array}{ll}
y_{1}(z) & y_{2}(z) \\
y_{1}^{\prime}(z) & y_{2}^{\prime}(z)
\end{array}\right]
$$

and

$\mathrm{Z}(z) \equiv\left[\begin{array}{ll}1 & 0 \\ z & 1\end{array}\right]\left[\begin{array}{cc}-\left(R_{2}(z) y_{1}^{\prime \prime}(z)\right)^{\prime}-R_{1}(z) y_{1}^{\prime}(z) & -\left(R_{2}(z) y_{2}^{\prime \prime}(z)\right)^{\prime}-R_{1} y_{2}^{\prime}(z) \\ R_{2}(z) y_{1}^{\prime \prime}(z) & R_{2}(z) y_{2}^{\prime \prime}(z)\end{array}\right]$

satisfy the system (3.21) with

and

$$
E(z)=\frac{1}{R_{2}(z)}\left[\begin{array}{rr}
z^{2} & -z \\
-z & 1
\end{array}\right]
$$

$$
-F(z)=\left[\begin{array}{ll}
R_{0}(z) & z R_{0}(z) \\
z R_{0}(z) & z^{2} R_{0}(z)-R_{1}(z)
\end{array}\right]+\left[\begin{array}{ll}
0 & R(z) \\
0 & z R(z)
\end{array}\right]
$$

Noting det $Y(z)=1 \cdot \operatorname{det}\left[\begin{array}{ll}y_{1} & y_{2} \\ y_{1}^{\prime} & y_{2}^{\prime}\end{array}\right]$ we conclude, if Theorem 3.6 holds, there exist double zero free solutions of (4.24) on $\Gamma$.

\section{Remarks}

(a) It is natural, in the context of this paper, to work with regular functions $\phi$ and $g$ in the scalar nonoscillation theorems. It is clear, however, from an inspection of the proofs, that a much broader class of functions could be used, say $C$ functions for $\phi$ and $C^{\prime}$ functions for $g$.

(b) It is no harder to consider, in place of (3.21), the system

$$
\begin{aligned}
& Y^{\prime}(z)=A(z) Y(z)+E(z) Z(z) \\
& Z^{\prime}(z)=-F(z) Y(z)+B(z) Z(z)
\end{aligned}
$$

and to obtain nonoscillation theorems involving a " $g$ " function. Indeed one would start with the substitution

$$
U(z) G^{-1}(z)=-Z(z) Y^{-1}(z)
$$

(where $G(z) \equiv g(z) I, g \neq 0$ on $\Gamma$ ), and proceed as above. 
(c) The last example of Section 4 can be generalised in an obvious manner to higher order equations and one can conclude the existence of solutions of equations of the form

$$
\sum_{k=0}^{n}\left(R_{k}(z) y^{(k)}(z)\right)^{(k)}+\sum_{k=0}^{n-1} p_{k}(z) y^{(k)}(z)=0,
$$

which are $n$th order zero free in domains of the $z$ plane.

(d) There are no restrictions on the symmetry of the $P$ matrix in Theorem 3.3 , nor any restrictions on the definiteness of the $E$ and $F$ matrices in Theorem 3.6 .

\section{REFERENCES}

(1) H. HeRoLd, Nullstellen bei Lösungen komplexer linearer Differentialgleichungen 2. Ordnung, Monatsh. Math. 74 (1970), 41-49.

(2) H. Herold, Nullstellen und kritische Stellender Lösunger von Differentialgleichungen 2. Ordnung im Komplexen, Math. Z. 108 (1969), 269-284.

(3) E. HrLle, Lectures on Ordinary Differential Equations (Addison-Wesley, 1969). 9-19.

(4) W. J. Кıм, Disfocality of Differential Systems, J. Math. Anal. Appl. 26 (1969),

(5) W. J. KIM, On the Zeros of $y^{(n)}+p y=0, J$. Math. Anal. Appl. 25 (1969), 189-208.

(6) M. LAVIE, OnDisconjugacy and Interpolation in the Complex Domain, J.Math. Anal. Appl. 32 (1970), 246-263.

(7) J. BARRETT, Fourth order boundary value problems and comparison theorems, Canad. J. Math. 13 (1961), 625-638.

(8) H. C. Howard, Some oscillation criteria for general self-adjoint differential equations, J. London Math. Soc. 43 (1968), 401-406, ibid (2) 1 (1969), 660.

UNIVERSITY OF KENTUCKY

LEXINGTON, KENTUCKY 40506

U.S.A. 